\title{
Elicitación de requisitos no funcionales basada en la gestión de conocimiento de los stakeholders
}

\author{
Non-functional requirements elicitation based on stakeholders's \\ knowledge management
}

\author{
Sandra L. Buitrón ${ }^{1 *} \quad$ Brenda L. Flores-Rios ${ }^{2} \quad$ Francisco J. Pino ${ }^{1}$ \\ Recibido 26 de abril de 2016, aceptado 13 de marzo de 2017 \\ Received: April 26, $2016 \quad$ Accepted: March 13, 2017
}

\begin{abstract}
RESUMEN
La Elicitación de Requisitos implica un proceso de entendimiento de las necesidades funcionales y las restricciones (Requisitos No Funcionales - RNF) establecidas por los stakeholders, las cuales serán entrada para las demás etapas del desarrollo de software. En dicha elicitación, la inclusión de los RNF depende de las estrategias para gestionar el conocimiento de los interesados (stakeholders y elicitadores) con el objetivo de generar productos software de alta calidad. Este documento se centra en establecer los diferentes flujos de transformación del conocimiento que se generan en el proceso de Elicitación de RNF al integrar diferentes componentes de la gestión del conocimiento. El núcleo de transformación del conocimiento propuesto fue valorado mediante las técnicas Focus Group y reporte de experiencia obteniendo resultados positivos para su implementación dentro de las prácticas de las organizaciones dedicadas al desarrollo de software.
\end{abstract}

Palabras clave: Ingeniería de software, elicitación de requisitos, gestión del conocimiento, requisitos no funcionales.

\begin{abstract}
Requirements elicitation involves a process of understanding of the functional requirements and restrictions (Non-Functional Requirements - NFR) established by the stakeholders, which will be input to the other stages of software development. In this elicitation, the inclusion of NFR depends on the strategies to manage knowledge of stakeholders (stakeholders and elicitors) in order to generate high-quality software products. This document focuses on establishing the different flows of transformation of knowledge generated in the NFR elicitation process by integrating different components of knowledge management - KM. The core of the proposed transformation knowledge was valued by Focus Group technique and reporting experience with positive results for its implementation within the practices of organizations dedicated to software development.
\end{abstract}

Keywords: Software Engineering, Requirements elicitation, Knowledge Management, Non- Functional Requirements.

\footnotetext{
1 Grupo IDIS. Universidad del Cauca. Facultad de Ingeniería Electrónica y Telecomunicaciones. Calle 5 No 4-70. Popayán, Colombia.E-mail: sandrabr@unicauca.edu.co, fjpino@unicauca.edu.co

2 Instituto de Ingeniería. Universidad Autónoma de Baja California. Calle de la Normal s/n y Blvd. Benito Juárez. Mexicali, B. C., México. E-mail: brenda.flores@uabc.edu.mx

* Autor de correspondencia
} 


\section{INTRODUCCIÓN}

El estándar IEEE 830-1998 indica que el proceso de Especificación de Requisitos (Software Requirements Specification) incluye, dentro de sus partes esenciales, información sobre interfaces externas, funciones, requisitos de desempeño, requisitos lógicos de base de datos, restricciones de diseño, atributos del software [1]. Así mismo, el proceso de Elicitación de Requisitos (ER) se refiere a todo sobre aprender y entender las necesidades de los usuarios y de los interesados del proyecto de software con el objetivo de comunicar estas necesidades a los desarrolladores del sistema [2]. De esta forma, el proceso de ER se considera como la base para que las etapas siguientes de un proceso de desarrollo de software plasmen de manera adecuada y completa la(s) alternativa(s) de solución [1-3].

Dentro de la ER, se toman en cuenta dos elementos principales: los Requisitos Funcionales (RF) y los Requisitos No Funcionales (RNF) [4]. Los RF son las acciones que debe realizar el software sin considerar las limitaciones físicas; mientas que los RNF definirán las propiedades ambientales y las restricciones de implementación relacionadas con el desempeño del producto software [3]. Para Chung, los RNF deben ser considerados como parte del proceso de desarrollo de software debido a que representan características de usabilidad, flexibilidad, desempeño, operatividad y seguridad del producto solicitado [4]. Por otro lado, se indica que los RNF limitan el comportamiento y el desarrollo de un producto software especificando los atributos que el sistema resultante debe tener [5]. Además, se señala que la funcionalidad está relacionada a lo que el sistema hace y su no funcionalidad o calidad se refiere a cómo el sistema se comporta frente a atributos observables como el desempeño, reusabilidad, confiabilidad [6]. Por todo lo anterior, los RNF deben considerarse, de manera determinante, en la definición de las restricciones tanto para el desarrollo como para el proceso de implementación [7] cumpliendo de manera completa las expectativas de los interesados (stakeholders y elicitadores) [8].

Este artículo se centra en el proceso de Elicitación de RNF debido a la importancia de identificar, analizar, documentar e integrar los RNF en el proceso de ER. De tal manera, que se logre un mayor cubrimiento y cohesión de estos requisitos con el diseño de la solución, y al mismo tiempo una mayor participación del stakeholder en el proceso de Elicitación de RNF en vista de que es un factor de éxito de éste proceso [9]. Por otro lado, el documento aborda la necesidad de gestionar el conocimiento relacionado a las necesidades del stakeholder frente a los RNF, así cómo utilizar los diversos tipos de conocimiento que poseen los elicitadores, de manera que se pueda resolver la pregunta: ¿Cómo apoyar el proceso de Elicitación de RNF (de aquí en adelante procesos de ERNF), dentro de las organizaciones, siguiendo un enfoque de Gestión de Conocimiento (GC)? Para dar respuesta a esta pregunta, en este artículo se presenta un núcleo de Transformación del Conocimiento entre stakeholders y elicitadores (denominado Núcleo TCER), para guiar el proceso de ERNF a través de componentes basados en el enfoque de GC. La relevancia de utilizar la GC se debe a que esta disciplina permite, mediante un proceso sistemático, crear y usar el conocimiento [10] representado como una mezcla de experiencia, valores, información y saber hacer que sirve como marco para la incorporación de nuevas experiencias e información, útil para la acción [11]. El núcleo TCER fue evaluado mediante expertos y a través de un reporte de experiencia obteniendo evidencia de que TCER puede apoyar la ERNF en procesos de desarrollo de productos software.

El documento se estructura de la siguiente manera: en la sección 2 se presenta el contexto de la GC y la ER, los trabajos relacionados y la metodología KoFI utilizada para la construcción del Núcleo TCER; la sección 3 detalla la implementación de cada de las fases de la metodología KoFI para la construcción del Núcleo TCER; la sección 4 analiza los resultados del proceso de evaluación con dos técnicas utilizadas y finalmente, en la sección 5 se presentan las conclusiones y actividades del trabajo futuro.

\section{FUNDAMENTOS}

\section{Gestión de conocimiento}

La Gestión del Conocimiento (GC) se define como un proceso sistemático para adquirir, organizar, nutrir, aplicar, compartir y renovar todas las formas de conocimiento, para mejorar la eficiencia organizacional y crear valor [11]. Así mismo, se refiere a todas las acciones para construir y establecer el conocimiento a través de entender cómo éste es creado, adquirido, procesado, distribuido, usado y 
controlado en las organizaciones [12]. Para gestionar el conocimiento, primero es necesario especificar que existen varios tipos de conocimiento, entre los que se resaltan el conocimiento tácito y el explícito [13]. El conocimiento tácito es el que se deriva de la experiencia personal y contiene cualidades intrínsecas que lo hacen difícil de articular, mientras que el conocimiento explícito se refiere al conocimiento que se expresa con palabras, números, símbolos y puede ser compartido fácilmente entre el equipo del proyecto [10].

Una estrategia que permite identificar las interacciones y flujos de conocimiento, es el modelo SECI (Figura 1), el cual a partir de la interacción dinámica y continua, entre el conocimiento tácito y explícito, se crea una espiral de conocimiento [10]. El objetivo del modelo SECI es brindar un entendimiento de cómo las organizaciones crean conocimiento para maximizar su administración, aplicación y transferencia por medio de los procesos de Socialización, Exteriorización, Combinación e Interiorización [13].

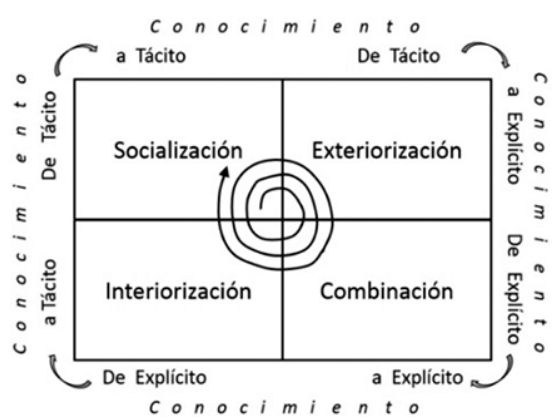

Figura 1. El modelo SECI (Adaptado de [13])

La aplicación de la GC en el campo de desarrollo de software ha sido de gran utilidad dada la naturaleza misma de la Ingeniería de Software como una disciplina intensiva en conocimiento [14]. El conocimiento en este campo juega un papel importante en la identificación de cómo la experiencia, lecciones aprendidas y mejores prácticas de desarrollos previos es interiorizada por los roles participantes y utilizada para mejorar la calidad de los productos [15].

Los flujos de conocimiento representan el atributo dinámico del conocimiento [16] y la transferencia del saber cómo, la cual consiste en un valor estratégico para la organización [17]. De esta forma, el intercambio de tipos de conocimiento entre los diferentes interesados, considerando de donde reside a dónde se necesita a través del tiempo, espacio y distribución geográfica es un camino previo necesario para dar inicio al proceso de desarrollo de software. Por tal motivo, el aporte de este trabajo es analizar, bajo el enfoque de GC, el proceso de ERNF desde las etapas tempranas de dicho proceso.

\section{Elicitación de Requisitos}

Se define la Elicitación de Requisitos (ER) como "la fase principal de un ciclo de desarrollo de software enfocada en recopilar y analizar los requerimientos y objetivos deseados para el sistema desde diferentes puntos de vista" [3]. Por ejemplo, los del cliente y stakeholders, las restricciones, el entorno de operación del sistema, necesidades de comercio, marketing y estándares, entre otros.

El proceso próximo a la ER se encuentra en la etapa de diseño del software; desde una perspectiva de arquitectura de software; se da valor y relevancia a la definición de los RNF. Al revisar la literatura, se detectaron dos propuestas desde la perspectiva de arquitectura de software donde se proponen alternativas de inclusión de estos RNF: i) The QualityDriven Software Re-engineering Framework, en el cual se describe cómo los RNF de desempeño y mantenibilidad son definidos a través del Framework para guiar el proceso de reingeniería de software a partir de un sistema legado [18], y ii) el enfoque Quality-Driven para representar RNF dentro de la arquitectura de software usando tácticas de arquitectura, las cuales permiten definir soluciones generales de arquitectura [19]. La investigación realizada por [20] sobre el estado del proceso de ERNF, permite comprobar que las técnicas utilizadas para realizar el proceso de ER sobre RNF no están aún claras. De la misma manera, se afirma que "aunque existen técnicas bien desarrolladas para obtener RF, existe una falta de mecanismo de ERNF y no existe un consenso adecuado al respecto de los RNF" [7].

\section{Trabajos relacionados}

Para el estudio de las propuestas acerca de la ERNF relacionada con el conocimiento y/o la gestión del mismo, se realizó un Mapeo Sistemático [21]. Primero, en el análisis se incluyó las revistas, 
memorias de congresos, memorias de simposios y reportes técnicos que mencionaban explícitamente la ERNF. Posteriormente, esta información fue filtrada aplicando el criterio de exclusión que permitió descartar documentos que no se centraran en la disciplina de GC. Se definieron tres dimensiones para el análisis de la información primaria publicada en los últimos diez años (periodo 2005-2015): Tipo de contribución, Tipo de validación utilizada y Forma en que integran la GC. Como resultado del Mapeo Sistemático se encuentran 37 propuestas de ERNF relacionadas con GC; en la Tabla 1 se presenta el subconjunto de las propuestas más recientes.

El Mapeo Sistemático permitió evidenciar la falta de contribuciones que integren la GC como fundamento para la realización del proceso de ERNF. De esta manera, este trabajo se centra en definir un Núcleo de Transformación del Conocimiento que guíe al proceso de ERNF con componentes basados en el enfoque de GC (denominado Núcleo TCER), desde las etapas tempranas de la ER. De esta forma, se desea brindar propuestas a la falta de participación activa de los stakeholders, apoyando en la definición clara de los RNF impactando en la mejora de las prácticas de desarrollo para alcanzar una mayor calidad en los productos software.

\section{Proceso metodológico}

La metodología utilizada para la construcción del núcleo TCER fue KoFI (Knowledge Flow Identification) [33]. Esta metodología define los lineamientos para la identificación y el análisis de flujos de conocimiento en los procesos organizacionales y se compone de las siguientes fases: i) especificar el proceso a analizar, ii) modelar el proceso con enfoque en flujos de conocimiento; iii) identificar flujos y tipos de conocimiento; y iv) identificar y analizar los flujos de conocimiento dentro de un grupo de trabajo. Cada una de las fases de KoFI se realiza como un proceso iterativo e incremental. La siguiente sección presenta el núcleo TCER, el cual fue estructurado siguiendo los pasos propuestos por la metodología KoFI.

\section{NÚCLEO DE TRANSFORMACIÓN DE CONOCIMIENTO EN ELICITACIÓN DE REQUISITOS - TCER}

\section{Fase 1: Especificar el proceso a analizar}

En reportes de Standish Groups's International se indican las tres razones principales que conllevan al fracaso de los proyectos de desarrollo de software: 1) con un $15.9 \%$ la falta de participación de los usuarios; 2) la falta de soporte a la gestión ejecutiva

Tabla 1. Resumen de propuestas del Mapeo Sistemático frente a ERNF relacionada con GC.

\begin{tabular}{|l|l|}
\hline \multicolumn{1}{|c|}{ Cómo se aborda el conocimiento } & \multicolumn{1}{c|}{ Fundamentación } \\
\hline $\begin{array}{l}\text { Niveles de conocimiento, el cual se combina e intercambia en un } \\
\text { contexto [22]. }\end{array}$ & Prototipado e intercambio de conocimiento. \\
\hline $\begin{array}{l}\text { La reutilización de RNF previamente definidos aumentan las alternativas } \\
\text { de requisitos a implementar [23]. }\end{array}$ & Ontología y Reutilización del conocimiento. \\
\hline $\begin{array}{l}\text { Existen dimensiones de calidad que se relacionan y descomponen para } \\
\text { lograr un afinamiento del conocimiento de los RNF [24]. }\end{array}$ & Jerarquía y Capas relacionadas. \\
\hline $\begin{array}{l}\text { Analizar los enfoques del proceso de Elicitación de RNF desde las } \\
\text { dimensiones: contexto, proceso y aplicación [25]. }\end{array}$ & $\begin{array}{l}\text { Análisis multidimensional de enfoques } \\
\text { existentes. }\end{array}$ \\
\hline $\begin{array}{l}\text { El Dominio del conocimiento permite inferir requerimientos que no } \\
\text { son explícitos [26]. }\end{array}$ & $\begin{array}{l}\text { Entendimiento de las necesidades de los } \\
\text { interesados. }\end{array}$ \\
\hline $\begin{array}{l}\text { Cualidades externas e internas del software. iQuién decide los RNF, } \\
\text { qué tipos de RNF importan? [27] }\end{array}$ & $\begin{array}{l}\text { Perspectiva de los arquitectos y cualidades } \\
\text { del software. }\end{array}$ \\
\hline $\begin{array}{l}\text { El conocimiento se intercambia al momento de la obtención de los } \\
\text { requisitos [28]. }\end{array}$ & $\begin{array}{l}\text { Facilitadores de flujos de conocimiento } \\
\text { y artefactos. }\end{array}$ \\
\hline Definición de requisitos de calidad (RNF) [29]. & Norma ISO 9126. \\
\hline $\begin{array}{l}\text { Toma en cuenta el conocimiento de los stakeholders para iniciar el } \\
\text { proceso de ER [30]. }\end{array}$ & $\begin{array}{l}\text { Trazabilidad de requisitos tempranos y } \\
\text { modelado visual. }\end{array}$ \\
\hline El conocimiento sobre los RNF está en el documento de especificación [31]. & Representación bajo metodología UML. \\
\hline El conocimiento se transfiere entre estados de colaboración [32]. & Perspectiva de la colaboración. \\
\hline
\end{tabular}


del proyecto representada con un $13.9 \%$; y 3) la definición clara de los requisitos con un $13 \%$ [34]. En este mismo estudio, se indica que los principales factores que generan los cambios en los proyectos de desarrollo de software son: con un $12,8 \%$ la falta de entradas de información del stakeholder, un $12,3 \%$ correspondiente a requisitos y especificaciones incompletas y un $11,8 \%$ por cambios en estos requisitos y especificaciones. De igual manera, el estudio confirma con un $13,1 \%$, que la causa principal por la cual los proyectos de desarrollo de software se deterioran y finalmente se cancelan, se debe a requisitos incompletos. Autores como [35] confirman que frente al objetivo de lograr la calidad en las organizaciones, un $37,5 \%$ de las no conformidades corresponde a RNF; entre otros datos resultado se identifica que $5,88 \%$ de las no conformidades hacen parte del proceso de definición de los requisitos de los stakeholders y un 12,94\% de las no conformidades se encuentran en el proceso de análisis de los requisitos del sistema. Estas estadísticas soportan y evidencian la importancia de una correcta y completa ejecución del proceso de ER y en mayor precisión, ER enfocada en los RNF. Para que el equipo de ER sea exitoso se requiere, de un profundo conocimiento del dominio de aplicación, Tecnologías de la Información y del proceso de elicitación [2]; es decir, una correcta combinación de tipos y fuentes de conocimiento, recursos y procesos.

\section{Fase 2: Modelo Conceptual del Núcleo TCER}

La metodología KoFI especifica que para analizar los flujos de conocimiento se requiere modelar el proceso de manera global y detallada. En el modelado global se identificó el núcleo TCER el cual se apoya en la GC para enmarcar una necesidad de definición conceptual de los RNF en el proceso de ER, en función de alguno de los siguientes escenarios:

- Los RNF son considerados importantes y necesarios para el proceso de desarrollo del producto de la organización desarrolladora de software

- Los RNF no son conocidos o no son aún concretos como formas claras de aplicación

- Los RNF no son entendidos por todos los involucrados en el proceso de Elicitación de Requisitos

- Los RNF no tienen el mismo significado para todos dentro de un contexto específico
El núcleo conceptual TCER contiene la dinámica de relaciones que se genera entre los grupos de interés (cliente externo y cliente interno, involucrados en el proceso de ERNF) quienes dentro de una cultura organizacional, y a través de múltiples canales de comunicación, generan un efecto de transformación del conocimiento guiados por una cultura de conocimiento organizacional. En esta, es importante el empleo del tiempo y recursos para comunicar los nuevos procedimientos de trabajo basados en el trabajo en grupo, la cooperación, la discusión y el análisis. La comunicación sirve también para interiorizar y crear la nueva cultura organizacional, flexible y creativa [36]. El propósito del núcleo TCER es integrar de manera recíproca y evolutiva el conocimiento de los RNF con los diversos tipos de stakeholders (usuario final, usuario de dirección, diseñador, arquitecto, desarrollador, administrador de base de datos, probadores, entre otros) involucrados dentro del proceso de ER.

En la Figura 2 se presenta cómo en forma paralela, el núcleo TCER soporta los conceptos y elementos relacionados con la GC enfocados al conocimiento tácito involucrado, tanto individual como colectivo, dentro del proceso de ERNF para un producto software.

Fase 3: Identificar fuentes y tipos de conocimiento Los stakeholders pueden apoyarse de diversas fuentes de conocimiento (roles, guías, plantillas, productos de trabajo, recursos o herramientas) que no sólo le ayuden a ejecutar sus actividades sino también a la toma de decisiones e incrementar sistemáticamente el conocimiento [33].

El elicitador (representado por un analista de sistemas, ingeniero de requisitos) posee conocimiento

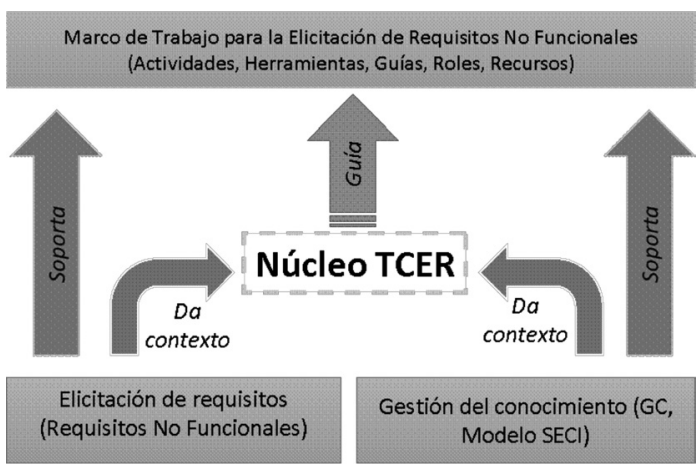

Figura 2. Modelo conceptual del núcleo TCER. 
tácito debido a su formación profesional y posible experiencia previa en el proceso de ERNF y cuenta con un entendimiento base acerca de este tipo de requisitos; mientras que el stakeholder puede desconocer u omitir estos aspectos durante el proceso de Elicitación.

En las organizaciones de software el conocimiento explícito se identifica en forma de procedimientos y procesos definidos, artefactos, bases de datos, archivos, código, normas o estándares, entre otros [33]; de esta forma, el documento de especificación de los RNF, representa el conocimiento explícito, debido a que describe los RNF a través de lenguaje natural y artefactos de apoyo, de manera que el stakeholder pueda validarlos y realimentar el conocimiento explícito descrito, logrando finalmente su aprobación. Esto significa que el elicitador es el actor principal en generar conocimiento explícito por medio del documento de especificación de los RNF, el cual podrá ser complementado con otro conocimiento explícito existente en otros documentos de ingeniería de requisitos o documentos de RNF de otros proyectos.

Fase 4: Identificar y Analizar flujos de conocimiento. El modelo SECI permite la creación, transferencia y conversión del conocimiento dentro de las organizaciones [13]. Por tal motivo, la GC permitirá aportar al proceso de ERNF en la consecución, análisis y definición de los RNF a través de la identificación de elementos como tipos y flujos de conocimiento. Durante la ejecución del proceso de ERNF se evidencian estos elementos en las actividades, en sus involucrados (stakeholders y elicitador) y en los productos de trabajo que se generan, buscando una mayor precisión para la calidad del producto software.

Un proceso de conocimiento es aquel proceso dinámico que se presenta entre los participantes (actores) de un proceso de conocimiento en un contexto donde el conocimiento se crea, transforma, propaga y aplica [16]. La Figura 3 muestra un modelo detallado de los componentes de TCER y cómo ocurre, a través de estos componentes, la transformación del conocimiento tácito frente a los RNF durante el proceso de ERNF. Bajo esta consideración es importante aclarar que TCER no describe un flujo de trabajo del proceso de ERNF, sino que éste núcleo es una representación de los eventos de transformación de conocimiento que se generan en éste proceso de elicitación de ERNF. A continuación, se describe estos aspectos de TCER.

El núcleo TCER define unos componentes base de la transformación, tales como: (i) el conocimiento de los stakeholders frente a conceptos de RNF, (ii) el conocimiento del elicitador frente a conceptos de RNF, (ii) el conocimiento explícito en documentos de especificación de otros proyectos. Así mismo, TCER cuenta con componentes dinamizadores de la transformación, tales como: (i) flujos de socialización, (ii) flujos de exteriorización, (iii) flujos de combinación y, (iv) flujos de interiorización, los cuales toman y transforman los componentes base durante la ejecución del proceso de elicitación. Estos flujos guían el proceso de ERNF apoyándose en los componentes técnicos de la transformación: (i) Elaboración de la especificación, y (ii) Validación de la especificación. Estos componentes técnicos son responsabilidades asignadas a los involucrados durante el proceso. Del mismo modo, TCER cuenta con componentes de soporte a la transformación, tales como: (i) la retroalimentación del conocimiento adquirido, (ii) la consolidación del conocimiento adquirido y (iii) el apoyo para completar la especificación de RNF.

Haciendo uso de los componentes de TCER, descritos en el párrafo anterior, el proceso de ERNF es ejecutado partiendo de la contextualización que realiza el elicitador a los stakeholders, mediante procesos altamente dinámicos de comunicación, acerca de qué son, la importancia, en qué consisten y las posibles maneras de identificar los RNF para el producto software a desarrollar. El dominio de conocimiento del elicitador permite que se identifiquen desde etapas tempranas del proceso de elicitación, así como reconocer su importancia y la necesidad de obtenerlos.

Un escenario de conocimiento, en esta contextualización, es cuando el conocimiento tácito del stakeholder, respecto a los RNF, es mayor que el conocimiento tácito del elicitador. A medida que se realiza esta transferencia de conocimiento tácito, el stakeholder adquiere nuevos significados o la modificación de significados previamente adquiridos, de manera que empieza a interiorizar los conceptos, importancia, propósitos y formas de identificar los RNF. Este escenario propicia la Interiorización del 


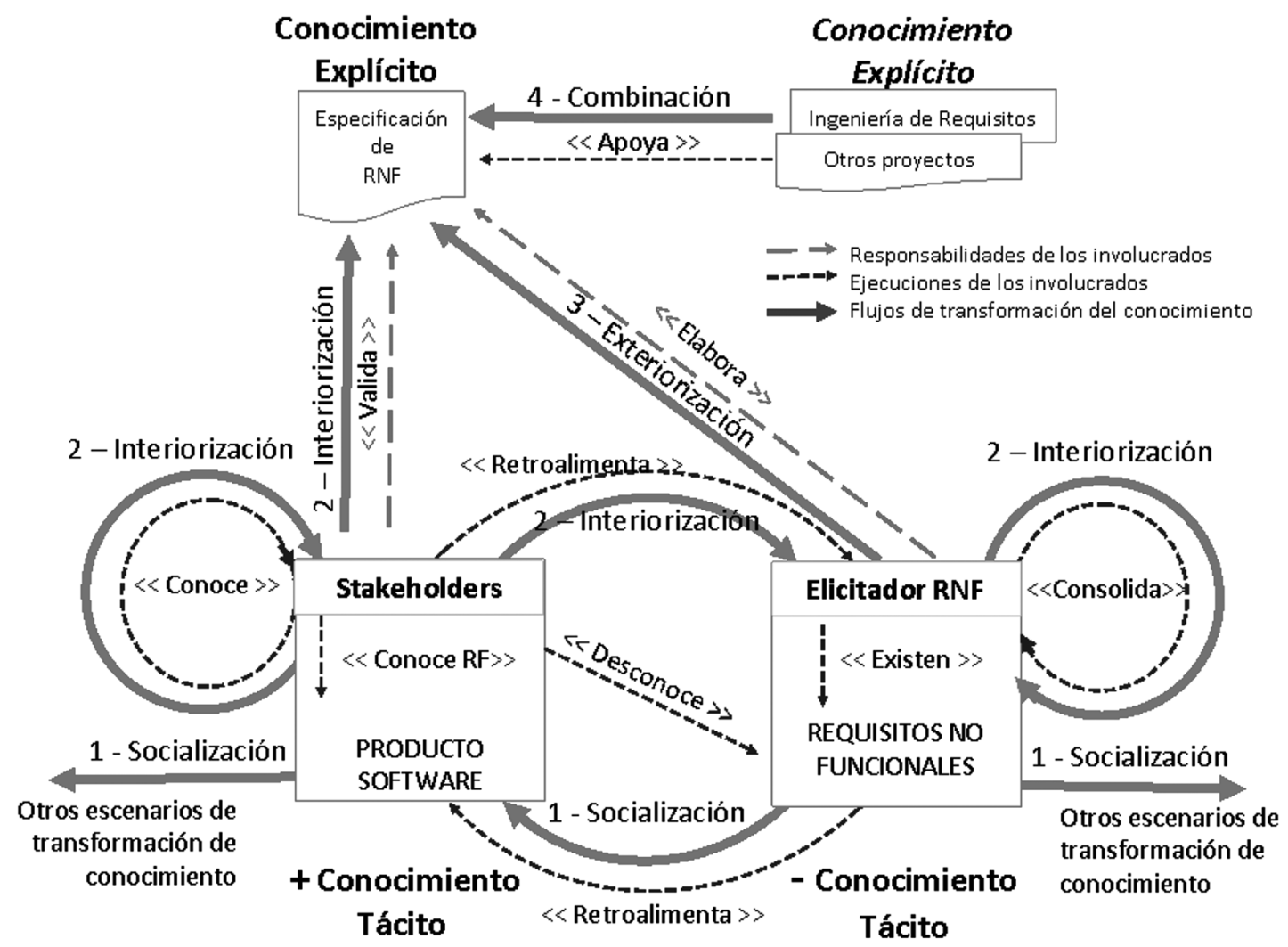

Figura 3. Núcleo TCER: Transformación del Conocimiento en el proceso de ERNF.

conocimiento explícito en el stakeholder. En esta dinámica, el elicitador también tiene la posibilidad de transformar su conocimiento tácito desde la perspectiva de ampliar y confirmar lo que entiende acerca de los RNF, debido a que expresa y explica las restricciones de manera que el stakeholder los identifique y comprenda. Así mismo, el elicitador utiliza la información obtenida para iniciar la concreción de estos requisitos (Interiorización del conocimiento en el elicitador), lo cual conlleva a representar, diagramar y redactar estos requisitos, propiciando el proceso de Exteriorización. El conocimiento exteriorizado, tanto por el elicitador como por el elicitado, permite aumentar el grado de precisión de los RNF, logrando finalmente, un conocimiento totalmente explícito entre los involucrados en el proceso.

Los procesos de creación y transferencia de conocimiento conllevan de manera inherente, a la generación de nuevos canales de comunicación y por lo tanto de formas de combinación y socialización, en las cuales se ven involucrados nuevos actores y recursos que a su vez tendrán ciertos niveles de conocimiento tácito o explícito frente a este tipo de requisitos. De acuerdo a esta dinámica individual y organizacional, la combinación y socialización son activadores de la interiorización y la exteriorización del conocimiento.

\section{EVALUACIÓN DEL NÚCLEO TCER}

El núcleo de transformación de conocimiento TCER fue evaluado de manera preliminar a través de dos mecanismos: i) Focus Group y ii) Reporte de experiencia. El mecanismo de Focus Group para poder obtener una retroalimentación inicial sobre la propuesta de TCER como núcleo del proceso de transformación de conocimiento en un proceso de ERNF y posteriormente, un reporte de experiencia que permitió aplicar TCER en proyectos de desarrollo de software, utilizando las mejoras que se habían identificado en el Focus Group. 


\section{FOCUS GROUP}

La presentación de los resultados del Focus Group se han estructurado en cuatro partes: i) La planeación de los procedimientos y herramientas; ii) el desarrollo de las evaluaciones; iii) el análisis de los resultados obtenidos; y por último, iv) las conclusiones del ejercicio.

\section{Evaluación a través de Focus Group}

En el contexto de la Ingeniería de software, uno de los mecanismos para la validación de procesos y propuestas es Focus Group, dado que permite obtener resultados rápidos a partir de la sinergia entre los participantes del grupo focal, así como compartir experiencias y puntos de vista diferentes frente a un tema central [37]. La estructura procedimental que se utilizó para el desarrollo del Focus Group es la descrita en [37], siguiendo las etapas de:

- Planteamiento de la investigación: se incluyeron elementos como protocolo, agenda e instrumentos para el debate.

- Diseño del grupo focal: se realizó la identificación y selección de los participantes del Focus Group tomando como criterios el conocimiento tácito enfocado a la experiencia y experticia en el proceso de ER, gestión de proyectos, y en procesos de recolección de requisitos desde la perspectiva del cliente.
- Conducción de las sesión: se realizó el proceso de recolección de información con el uso de elementos como diapositivas para la presentación del núcleo TCER, material impreso para cada experto y grabadora de sonidos.

- Análisis de información y resultados: Se analizó la información recolectada para presentar datos cualitativos y cuantitativos resultantes de la evaluación.

\section{Desarrollo del Focus Group}

Etapa de Planteamiento de la Investigación: Se elaboró un resumen descriptivo del núcleo TCER, compartido a los participantes apoyando la contextualización y generación de ideas, antes y durante el desarrollo de la sesión. Se utilizó un formato de evaluación del núcleo TCER, de manera que cada participante registró los puntos positivos y los puntos de mejora a tomar en cuenta para su análisis y ajustes. La calificación del núcleo TCER frente a los tres criterios de evaluación se indica en la Tabla 2.

Etapa de Diseño del Grupo Focal: El criterio para la selección de los participantes del Focus Group fue la experticia (conocimiento tácito) frente al proceso de ER. De esta manera, el involucramiento de los participantes debía asegurar un rango mínimo de experiencia realizando este proceso en un contexto real y práctico. El grupo de expertos se formó por

Tabla 2. Criterios de evaluación del Núcleo TCER.

\begin{tabular}{|c|c|c|}
\hline Criterio de evaluación & Interpretación & Escala (De 1 a 5) \\
\hline $\begin{array}{l}\text { Nivel de claridad sobre el proceso } \\
\text { de transformación del conocimiento }\end{array}$ & $\begin{array}{l}\text { ¿Qué tan entendible es la trans- } \\
\text { formación del conocimiento en el } \\
\text { proceso de la elicitación de RNF? }\end{array}$ & $\begin{array}{l}\text { 1. No es entendible } \\
\text { 2. Poco entendible } \\
\text { 3. Medianamente entendible } \\
\text { 4. Entendible } \\
\text { 5. Muy entendible }\end{array}$ \\
\hline $\begin{array}{l}\text { Aumento del grado de explicites } \\
\text { sobre RNF }\end{array}$ & $\begin{array}{l}\text { ¿El núcleo TCER permite aumentar } \\
\text { la explicites de los RNF dentro del } \\
\text { proceso de elicitación de RNF? }\end{array}$ & $\begin{array}{l}\text { 1. No aumenta } \\
\text { 2. Aumenta muy poco la explicites } \\
\text { 3. Aumento medio de la explicites } \\
\text { 4. Aumenta la explicites } \\
\text { 5. Aumento sustancial de la explicites }\end{array}$ \\
\hline $\begin{array}{l}\text { Fortalecimiento del } \\
\text { entendimiento acerca de RNF }\end{array}$ & $\begin{array}{l}\text { ¿El núcleo TCER fortalece el } \\
\text { entendimiento de lo que son los } \\
\text { RNF, desde la perspectiva de la } \\
\text { gestión del conocimiento? }\end{array}$ & $\begin{array}{l}\text { 1. No fortalece el entendimiento } \\
\text { 2. Lo fortalece muy poco } \\
\text { 3. Fortalece en poca medida el } \\
\text { entendimiento } \\
\text { 4. Fortalece el entendimiento } \\
\text { 5. Fortalece en gran medida el } \\
\text { entendimiento }\end{array}$ \\
\hline
\end{tabular}


6 profesionales de Colombia y México con los perfiles que muestra la Tabla 3.

\section{Etapa de Conducción de la Sesión y Resultados:} La sesión de Focus Group se condujo acorde al protocolo definido bajo una dinámica de discusión y realimentación de ideas, ventajas, desventajas y opiniones acerca del núcleo TCER en la elicitación de RNF basada en GC. Toda la sesión fue grabada en audio para su revisión y análisis. Posterior a la discusión, se solicitó el diligenciamiento del formato de evaluación con el fin de capturar, en términos cuantitativos, las medidas iniciales acerca de la validez y buen entendimiento de TCER.

Etapa de Análisis de Información: En esta etapa se realizaron las siguientes actividades: i) Revisión de la información recolectada en audio, ii) Categorización de los comentarios como puntos de mejora/sugerencias/inclusiones y puntos positivos
(Tabla 4), y iii) Análisis de la información obtenida a través del diligenciamiento del formato de calificación (Tabla 5).

En la Tabla 2, se indican las interpretaciones de las calificaciones asignadas (en una escala de 1 a 5) para los tres criterios de evaluación. En la Tabla 5 se muestran estos resultados, donde el criterio que obtuvo el mayor número de calificaciones, con valor 5 , fue el que evalúa al Núcleo TCER como instrumento de GC que fortalece el entendimiento de los RNF; con una calificación promediada de 4.6. TCER obtuvo una calificación de 4.3 frente a su aporte sustancial para generar conocimiento más explícito de estos requisitos (segundo criterio de evaluación). Del mismo modo, el proceso de evaluación a través del Focus Group permitió conocer que el núcleo TCER requiere un mayor grado de detalle de manera que sea más entendible para los involucrados en el proceso de elicitación de RNF.

Tabla 3. Perfiles de profesionales participantes en Focus Group.

\begin{tabular}{|l|l|l|c|}
\hline \multicolumn{1}{|c|}{ Id } & \multicolumn{1}{|c|}{ Perfil } & \multicolumn{1}{c|}{ Características del perfil } & Total \\
\hline ER & $\begin{array}{l}\text { Experto en Elicitación } \\
\text { de Requisitos (ER) }\end{array}$ & $\begin{array}{l}\text { Profesionales de la ingeniería de sistemas con mínimo 7 años de } \\
\text { experiencia consecutiva, realizando el proceso de ER en organizaciones } \\
\text { bajo estructuras de desarrollo de software. }\end{array}$ & 4 \\
\hline U & $\begin{array}{l}\text { Experto en procesos } \\
\text { de negocio }\end{array}$ & $\begin{array}{l}\text { Profesionales con conocimientos en procesos de negocio con mínimo } \\
5 \text { años de experiencia. }\end{array}$ & 1 \\
\hline PMP & $\begin{array}{l}\text { Experto en gestión de } \\
\text { proyectos tecnológicos }\end{array}$ & $\begin{array}{l}\text { Profesional en gestión de proyectos con mínimo 8 años de experiencia } \\
\text { en administración de procesos de desarrollo de software. }\end{array}$ & 1 \\
\hline
\end{tabular}

Tabla 4. Consolidado y categorización de los resultados del Focus Group

\begin{tabular}{|c|c|}
\hline Puntos de mejora del núcleo TCER & Puntos positivos del núcleo TCER \\
\hline $\begin{array}{l}\text { 1. Profundizar en la socialización incluyendo los costos } \\
\text { adicionales para los RNF. } \\
\text { 2. Tomar en cuenta tipos de usuario, niveles de } \\
\text { conocimiento de los usuarios y tipos de requisitos } \\
\text { (externos, de negocio y de producto). }\end{array}$ & $\begin{array}{l}\text { 1. Permite al usuario final conocer sobre los RNF. } \\
\text { 2. Permite tomar en cuenta cosas que implican un } \\
\text { mayor presupuesto por parte del grupo de tecnología. } \\
\text { 3. Los procesos de socialización e interiorización } \\
\text { permiten transferir tipos de conocimiento. }\end{array}$ \\
\hline $\begin{array}{l}\text { 3. Definir formas concretas de llevar a cabo la } \\
\text { transformación del conocimiento. } \\
\text { 4. Revisar el núcleo para organizaciones que son casa } \\
\text { de software y para las que no lo son. } \\
\text { 5. Clasificar y seleccionar los RNF que están asociados } \\
\text { directamente al conocimiento del usuario final. } \\
\text { 6. Incluir una lista de preguntas/chequeo para manejar como } \\
\text { banco de conocimiento para el proceso de elicitación. } \\
\text { 7. Definir roles y responsabilidades del nivel de } \\
\text { dirección de los proyectos para asegurar que los } \\
\text { RNF se definan en los proyectos. }\end{array}$ & $\begin{array}{l}\text { 4. Permite dejar evidencia de recomendaciones dadas } \\
\text { por los ingenieros de requisitos hacia los usuarios } \\
\text { finales. } \\
\text { 5. El proceso de socialización permite asegurar la } \\
\text { inclusión de los RNF en los proyectos. } \\
\text { 6. Los RNF llegan a ser más críticos que los mismos } \\
\text { RF porque impactan todo el proyecto. } \\
\text { 7. Permite aumentar el grado de conciencia de los } \\
\text { usuarios finales sobre la importancia de los RNF en } \\
\text { las organizaciones y sus inversiones en tecnología. }\end{array}$ \\
\hline
\end{tabular}


De esta manera, se confirma la posibilidad de que el núcleo TCER sea adoptado por los proyectos de desarrollo de software que buscan mejorar la calidad de sus productos.

Tabla 5. Calificación de criterios de evaluación del núcleo TCER.

\begin{tabular}{|c|c|c|c|}
\hline $\begin{array}{l}\text { Criterios / } \\
\text { Evaluadores }\end{array}$ & 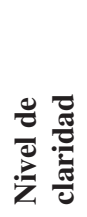 & 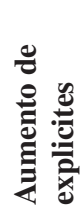 & 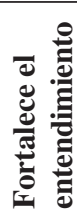 \\
\hline Evaluador 1 (ER) & 4 & 4 & 5 \\
\hline Evaluador 2 (ER) & 3 & 4 & 5 \\
\hline Evaluador 3 (ER) & 3 & 4 & 5 \\
\hline Evaluador 4 (ER) & 4 & 5 & 5 \\
\hline Evaluador $5(\mathrm{U})$ & 3 & 4 & 4 \\
\hline Evaluador 6 (PMP) & 5 & 5 & 4 \\
\hline Promedio & 3,6 & 4,3 & 4,6 \\
\hline
\end{tabular}

Luego de realizar el Focus Group se puede concluir que: (i) Debido a que TCER tiene como base la GC permite a los stakeholders involucrados en un proceso de ERNF tener una nueva perspectiva de aporte a los procesos de ingeniería de software desde su propia visibilidad, frente a los RNF. (ii) TCER es una propuesta que agudiza el uso de la GC como base para la mejora de procesos de ingeniería de software, así mismo como puede llegar a ser adaptable a los niveles de madurez de las organizaciones frente a sus procesos de desarrollo de software, (iii) TCER requiere ser detallado y descrito de manera más clara para que pueda ser entendido y llevado a la práctica por los usuarios, a través de la implementación de artefactos e instrumentos. En este sentido, TCER está siendo robustecido para que gane claridad a través de la adición y estructuración de componentes tanto técnicos como de GC, tales como: niveles de conocimiento de los stakeholders, tipos de stakeholders (caracterización e involucrados), categorías de los RNF, políticas y lineamientos organizacionales en el proceso de ERNF, escenarios de aplicación, tipos de flujos de conocimiento y grados de transformación del conocimiento (estos elementos están fuera del alcance de este articulo y se presentan en [38]). (iv) El uso habitual de TCER dentro de las organizaciones podría permitir un mayor grado de explicites y dominio de los RNF al interior de las mismas, de manera que aporta como mecanismo de mejora del proceso de desarrollo de software y de la calidad del producto software. (v) TCER integra los procesos de ingeniería de requisitos, el dominio de los stakeholders, la tecnología y la GC, entre quienes se genera un primer grado de cohesión/convergencia único y propio de cada organización, y (vi) TCER aporta de manera positiva a las prácticas de ERNF dentro de las organizaciones desde diversas perspectivas, tales como: los procesos de elicitación más completos, el aumento del conocimiento de los RNF, mejorar la planeación presupuestal, entre otros.

\section{Reporte de experiencia}

A continuación, se describe el reporte de experiencia del uso de TCER en una organización de desarrollo de software. La experiencia es descrita a través de: i) un contexto de aplicación, ii) la descripción de la organización participante, iii) un reporte y análisis del uso del núcleo, de acuerdo a la estructura propuesta por [39] y [40].

Contexto de aplicación: El núcleo TCER ha sido usado en los procesos de ER de una MyPyme colombiana, con el fin de descubrir los RNF para el desarrollo de productos software. Este proceso de elicitación se llevó a cabo con los diferentes stakeholders (usuarios finales y usuarios técnicos) de tres proyectos de desarrollo. Un primer proyecto (P1): Desarrollo de una plataforma para el control de inventarios, como producto propio de la organización, un segundo proyecto (P2): Desarrollo de un componente para gestión de indicadores de sostenibilidad de un sistema de acueducto y alcantarillado, y un tercer proyecto (P3): Desarrollo de un sistema de información para la gestión de acciones populares, el cual pretende controlar el registro y estado de los procesos jurídicos llevados a cabo por un área jurídica. La aplicación de TCER fue guiada por un investigador no intrusivo (quien es el primer autor) con el objetivo de generar la especificación de RNF a partir del uso de los flujos de conocimiento que se presentan en el proceso de ERNF de la organización, de manera que se obtenga evidencia de si TCER respalda el proceso de ERNF.

Características de la organización: La organización colombiana en la cual se realizó la experiencia de uso de TCER se llama E-TEKNIK, constituida hace 3 años con el propósito de brindar servicios especializados 
de desarrollo de soluciones tecnológicas dirigidas principalmente al mercado empresarial y académico. La empresa E-TEKNIK cuenta con 12 empleados actualmente quienes desempeñan roles de: i) líder de proyectos, ii) analista de requisitos, iii) diseñador, iv) desarrollador y v) probador de soluciones. Entre los productos destacados y comercializados de la organización se encuentran: una Plataforma para el manejo de información académica, que facilita a los docentes las actividades diarias de calificación, una Plataforma web donde se integran banco de proyectos, procesos administrativos e indicadores de gestión; articulados con la norma ISO9001 y MECI, y un producto para apoyar el proceso pedagógico desarrollando objetos virtuales de aprendizaje soportados en la realidad aumentada y realidad virtual, entre otros productos desarrollados a la medida.

Reporte y análisis del trabajo ejecutado en la organización: El uso de TCER permitió obtener información frente a la utilización de los flujos de conocimiento que se representan en este núcleo cuando se realiza un proceso de ERNF:

- El flujo de socialización se evidenció al momento en que el investigador impartió una capacitación, preliminar al uso de TCER, sobre los RNF a los líderes de los proyectos y parte de los integrantes del equipo técnico. Los stakeholders finales (usuarios de operación y jefes de áreas o procesos de negocio) no fueron socializados. Luego de la socialización se evaluó qué tanto conocimiento tácito habían ganado los participantes de la capacitación frente a RNF, y de acuerdo a los resultados (Tabla 6) los participantes ganaron entre el $30 \%$ y el $70 \%$ de conocimiento tácito acerca de RNF; conocimiento que previo a la socialización tenía porcentajes mínimos en cada participante.

Tabla 6. Rangos de conocimiento ganado luego de socialización de RNF.

\begin{tabular}{|l|l|l|l|l|l|}
\hline \multirow{2}{*}{$\begin{array}{c}\text { Rango de conocimiento ganado } \\
\text { luego de socialización }\end{array}$} & \multicolumn{5}{|c|}{ Participantes } \\
\cline { 2 - 6 } & $\mathbf{1}$ & $\mathbf{2}$ & $\mathbf{3}$ & $\mathbf{4}$ & $\mathbf{5}$ \\
\hline Entre 0 - 30\% de conocimiento & & & & & \\
\hline Entre 30\% y 50\% de conocimiento & & $\mathrm{x}$ & & $\mathrm{x}$ & $\mathrm{x}$ \\
\hline Entre 50\% y 70\% de conocimiento & $\mathrm{x}$ & & $\mathrm{x}$ & & \\
\hline Entre 70\% y $100 \%$ de conocimiento & & & & & \\
\hline
\end{tabular}

- El flujo de exteriorización se evidenció a través de la elaboración de las especificaciones de los RNF, los cuales quedaron explícitos para cada proyecto permitiendo así lograr el objetivo principal de un proceso de ERNF, como es la especificación de los RNF. Los RNF fueron modelados haciendo uso de plantillas de especificación que usaron como elementos principales los conceptos de: (i) flujo del proceso de negocio, para ubicar los RNF dentro de contextos de negocio más pequeños y que hacían parte del dominio de las aplicaciones, (ii) características de la ISO 25010, y (iii) interfaces: manuales, semi-automáticas o automáticas identificadas, (iv) requisitos legales entre otros.

- Los flujos de combinación e interiorización se evidenciaron de manera permanente a través de la realización de consultas planteadas por los elicitadores al investigador cuando tenían alguna inquietud acerca de la manera en que estaban llevando a cabo el proceso de identificación y especificación de RNF. El investigador participó aclarando dudas acerca de conceptos relacionados con RNF y los componentes de TCER, siendo un facilitador para el trabajo que desarrollaron de manera iterativa en los proyectos. De esta manera, los elicitadores demostraron a través de su interiorización el logro de un mayor entendimiento y dominio de los conceptos sobre RNF refinando los artefactos involucrados en el proceso.

A continuación, se describe el esfuerzo realizado por los participantes durante la aplicación del núcleo TCER en la organización. El primer esfuerzo que se requirió para posibilitar la aplicación de TCER fue la contextualización de la propuesta, en la cual participaron: (i) el gerente de la organización y, (ii) dos líderes de proyectos. Esta actividad implicó un total de 8 horas/hombre, incluyendo el esfuerzo del investigador, en una sola sesión con duración de 2 horas. Como resultado de esta actividad se definió los proyectos a los cuales se les aplicaría TCER. A partir de esta definición, en una segunda sesión, se realizó la capacitación técnica a la cual asistieron 5 ingenieros de sistemas quienes hacían parte de los equipos de desarrollo de los tres proyectos elegidos. La sesión tuvo una duración de 3 horas, para un total de esfuerzo de 18 horas/hombre del equipo investigador. Posterior a esta fundamentación acerca de RNF se iniciaron las actividades de elaboración 
de la especificación de RNF por cada uno de los elicitadores de los proyectos. Para esta actividad de especificación de RNF, haciendo uso de la plantilla respectiva de la propuesta, el proyecto (P1) requirió un esfuerzo de 16 horas/hombre, esfuerzo que fue limitado por restricciones de tiempo del proyecto, el proyecto $(\mathrm{P} 2)$ requirió 40 horas/hombre, esfuerzo que implicó reingeniería debido a que el proyecto ya estaba en etapa de entrega del producto software al momento de la intervención, y el proyecto (P3) requirió 6 horas/hombre a la especificación de los RNF, para un total de esfuerzo en especificación de RNF de 62 horas/hombre. En paralelo a la elaboración de la especificación de RNF en los proyectos, se ejecutaron 4 sesiones, de 2 horas cada una, para atender dudas de elicitación y uso de la plantilla de especificación; estas actividades requirieron un esfuerzo total de 32 horas del equipo investigador. Finalmente, en la sesión de cierre de la intervención a la empresa y sus proyectos, se requirió un esfuerzo de 12 horas/hombre del equipo investigador, con una duración de 2 horas en esta sesión. De acuerdo a estos datos, el esfuerzo total requerido por el grupo investigador, para los tres proyectos, para la aplicación de TCER fue de 132 horas/hombre. Otros esfuerzos realizados para la aplicación de TCER en la organización fueron los logísticos, impresión de plantillas, preparación del material de las capacitaciones, entre otros, propios de un proceso de replicación/capacitación de grupos.

Discusión: A partir del trabajo llevado a cabo en la organización, mediante la utilización de TCER, los participantes expresaron que: (i) una vez aplicada la propuesta de transformación de conocimiento en su proceso de elicitación de requisitos les permitió reconocer la importancia de los RNF para el desarrollo de software con calidad, y (ii) para futuros proyectos de desarrollo de software tendrán en cuenta la propuesta con el fin de fortalecer la generación de una especificación en pro de favorecer las etapas siguientes del desarrollo considerando los diferentes stakeholders de la especificación.

El investigador confirma que en el proceso de ERNF se generan procesos de transformación de conocimiento importantes los cuales apoyan la instauración de los RNF, por medio de los flujos de socialización e interiorización en los equipos de desarrollo, y de los de exteriorización y combinación que se reflejan en la especificación. Por otro lado, el uso de TCER en estos proyectos permitió obtener un total de $132 \mathrm{RNF}$ entre los tres proyectos (Tabla 7). Este número de RNF es relevante frente a la ausencia de RNF tomados en cuenta por la organización para sus desarrollos, hasta antes del uso de TCER en sus procesos de ERNF.

Tabla 7. Requisitos No Funcionales identificados con TCER por proyecto.

\begin{tabular}{|l|r|r|r|c|}
\hline Requisitos No Funcionales & P1 & P2 & P3 & Totales \\
\hline RNF Eficiencia de desempeño & 5 & 20 & 10 & 35 \\
\hline RNF Seguridad & 2 & 18 & 7 & 27 \\
\hline RNF Fiabilidad & 5 & 10 & 8 & 23 \\
\hline RNF Mantenibilidad & 0 & 0 & 1 & 1 \\
\hline RNF Usabilidad & 8 & 15 & 23 & 46 \\
\hline Totales por proyecto & 20 & 63 & 49 & 132 \\
\hline
\end{tabular}

Como activos de conocimiento relevantes, se identificaron las siguientes lecciones aprendidas: (i) para la aplicación de TCER se requiere, previo a su uso, una capacitación al equipo que cumplirá el rol de elicitador de manera que reconozca e identifique los RNF, las características y sub características de calidad que se buscan considerar en el proceso de elicitación, y (ii) el logro de obtener en menor o mayor medida los RNF en los proyectos de desarrollo de software depende de la experiencia y el conocimiento tácito de los participantes en el proceso de elicitación de requisitos. Este conocimiento tácito, acerca de los RNF, es viable de potencializarlo con TCER y por tanto su uso permite que la transformación del conocimiento se dé de manera natural dentro de la dinámica propia del proceso de ERNF. Estos resultados evidencian que TCER puede ser una herramienta que apoya el proceso de ERNF de manera práctica en las organizaciones desarrolladoras de software.

\section{CONCLUSIONES}

La ausencia de los procesos de conocimiento que transformen el conocimiento tácito (experiencia) a explicito, y viceversa, de los RNF (al no solicitarlos o no evaluar cuáles deberán implementarse), entre stakeholders y elicitadores, eleva el riesgo de elaboración de diseños de baja calidad, los cuales seguirán siendo definidos sólo bajo las necesidades funcionales. La revisión de la literatura llevada a 
cabo confirma la importancia de realizar mayores contribuciones en el área de ERNF debido a la necesidad de que las organizaciones brinden mecanismos prácticos que faciliten su inclusión dentro de sus procesos de desarrollo de software. Adoptar la GC, como enfoque base para los procesos de ERNF, conduce a las organizaciones a transformar sus tipos de conocimiento en un activo tangible administrable y por tanto planeado, estructurado y controlado; además de permitirles avanzar frente al objetivo de lograr una calidad medible de sus productos software.

La contribución principal de este trabajo, en pro de estas ventajas organizacionales, es el núcleo TCER el cual agudiza el uso del enfoque de GC como estrategia viable para que la alta dirección sea responsable de propiciar mecanismos para la creación, uso, salvaguardia y accesibilidad del nuevo conocimiento. Los procesos de conocimiento íntrinsecos del núcleo TCER favorecen directamente el flujo y la transformación del conocimiento tácito y explícito existentes entre los involucrados. Con TCER, los stakeholders tienen una nueva perspectiva de cómo aportar, desde su propio conocimiento tácito, a los procesos de la Ingeniería de Software. Específicamente, el despliegue de TCER dentro de las prácticas organizacionales, en desarrollo de software, permitirá un mayor grado de explicites y dominio de los RNF al interior de las mismas. Los componentes de GC que constituyen el núcleo TCER permiten su adaptación y aplicabilidad a otros contextos (por ejemplo otros procesos de la Ingeniería de Software) generando un escalamiento en los niveles de madurez de las organizaciones frente a sus áreas técnicas y reduciendo las brechas de conocimiento entre stakeholders e ingenieros de requisitos. Por otro lado, la validación preliminar de TCER a través de un reporte de experiencia, permite concluir que TCER es aplicable al proceso de ERNF generando resultados positivos frente al logro de la identificación de los RNF para productos software que serán desarrollados y al mismo tiempo se evidencía que TCER permite: (i) tener un proceso de elicitación más completo, y (ii) aumentar el conocimiento de los RNF por parte de los involucrados en el proceso de desarrollo (elicitador y usuarios). De esta forma, TCER permite la integración entre los procesos de ingeniería de requisitos, el dominio de los stakeholders, la tecnología y la GC, quienes en conjunto convergen hacia lograr un mejor producto software y a su vez a aumentar su calidad desde la mejora del proceso de ERNF.

Por último, se esta trabajando en la construcción de artefactos para un marco de trabajo, guíado por TCER, que permitan instrumentalizarlo haciendo uso de: (i) modelos de referencia de procesos relacionados con la gestión del conocimiento, tal como los propuestos por [41] y [42], y (ii) un modelo de evaluación de procesos de software como ISO 15504 , entre otros.

\section{AGRADECIMIENTOS}

Este trabajo ha sido financiado por el proyecto VRI4354 registrado en la Vicerrectoría de Investigaciones de la Universidad del Cauca y está asociado al proyecto 111/942 registrado en la Coordinación de Posgrado e Investigación de UABC. Sandra L. Buitrón y Francisco J. Pino agradecen a la Universidad del Cauca, donde ellos trabajan como profesores OTC y Titular respectivamente. Así mismo se agradece de manera especial al equipo de E-TEKNIK, a su gerente, a sus líderes de proyectos e ingenieros participantes en la experiencia.

\section{REFERENCIAS}

[1] I.C.S.S.E.S. Committee and I.-S.S. Board. "IEEE Recommended Practice for Software Requirements Specifications". 1998. Institute of Electrical and Electronics Engineers

[2] H.F. Hofmann y F. Lehner. "Requirements engineering as a success factor in software projects". IEEE software. Vol. $18 \mathrm{~N}^{\circ} 4$, pp. 58. 2001.

[3] D. Pandey, U. Suman and A. Ramani. "An effective requirement engineering process model for software development and requirements management". In Advances in Recent Technologies in Communication and Computing (ARTCom), 2010 International Conference on, pp. 287-291. IEEE. 2010.

[4] L. Chung and J.C.S. do Prado Leite. "On non-functional requirements in software engineering". In Conceptual modeling: Foundations and applications, ed: Springer, pp. 363-379. 2009.

[5] A. Casamayor, D. Godoy and M. Campo, "Identification of non-functional requirements in textual specifications: A semi-supervised 
learning approach". Information and Software Technology. Vol. $52 \mathrm{~N}^{\circ}$ 4, pp. 436-445. 2010.

[6] X. Franch and P. Botella. "Putting nonfunctional requirements into software architecture". In Proceedings of the 9th international Workshop on Software Specification and Design, Mie, Japan, p. 60. IEEE Computer Society. 1998.

[7] M. Rahman and S. Ripon. "Elicitation and modeling non-functional requirements-a POS case study". International Journal of Future Computer and Communication. Vol. $2 \mathrm{~N}^{\circ} 5$, pp. 485-489. 2014.

[8] L.M. Cysneiros and E. Yu. "Non-functional requirements elicitation". In Perspectives on software requirements. Ed: Springer, pp. 115-138. 2004.

[9] K. El Emam and N.H. Madhavji. "A field study of requirements engineering practices in information systems development". In Requirements Engineering. 1995. Proceedings of the Second IEEE International Symposium on, pp. 68-80. IEEE. 1995.

[10] I. Nonaka and D.J. Teece. "Managing industrial knowledge: creation, transfer and utilization”. Sage, 2001.

[11] T. H. Davenport and L. Prusak. "Working knowledge: How organizations manage what they know". Harvard Business Press. 1998.

[12] K.M. Wiig, R. de Hoog and R. Van Der Spek. "Supporting knowledge management: a selection of methods and techniques". Expert systems with applications. Vol. 13 $\mathrm{N}^{\circ}$ 1, pp. 15-27. 1997.

[13] I. Nonaka, R. Toyama and N. Konno. "SECI, Ba and leadership: a unified model of dynamic knowledge creation". Long range planning. Vol. $33 \mathrm{~N}^{\mathrm{o}}$ 1, pp. 5-34. 2000.

[14] J. Capote, C.J. Llantén, C. Pardo, A.J. González and C.A. Collazos. "Gestión del conocimiento como apoyo para la mejora de procesos software en las micro, pequeñas y medianas empresas". Ingenieria e investigacion. Vol. $28 \mathrm{~N}^{\circ}$ 1, pp. 137-145. 2008.

[15] T. Dingsøyr and R. Conradi. "A survey of case studies of the use of knowledge management in software engineering". International journal of software engineering and knowledge engineering. Vol. $12 \mathrm{~N}^{\circ} 04$, pp. 391-414. 2002.
[16] J. Guo and Y. Wang. "Context modeling for knowledge flow". In IEEE International Conference on Information Reuse and Integration. Las Vegas, NV, USA, pp. 330335. IEEE. 2008.

[17] A. K. Gupta and V. Govindarajan. "Knowledge flows within multinational corporations". Strategic management journal. Vol. $21 \mathrm{~N}^{\circ} 4$, pp. 473-496. 2000.

[18] L. Tahvildari, K. Kontogiannis and J. Mylopoulos. "Quality-driven software re-engineering". Journal of Systems and Software. Vol. $66 \mathrm{~N}^{\circ} 3$, pp. 225-239. 2003.

[19] S. Kim, D.-K. Kim, L. Lu and S. Park, "Quality-driven architecture development using architectural tactics". Journal of Systems and Software, Vol. $82 \mathrm{~N}^{\circ}$ 8, pp. 12111231. 2009.

[20] É. Serna-Montoya. "Estado actual de la investigación en requisitos no funcionales". Ingeniería y Universidad. Vol. $16 \mathrm{~N}^{\circ} 1$, pp. 225-246. 2012.

[21] K. Petersen, R. Feldt, S. Mujtaba and M. Mattsson. "Systematic mapping studies in software engineering". In 12th international conference on evaluation and assessment in software engineering, pp. 1-12. 2008.

[22] L. Teixeira, V. Saavedra, C. Ferreira, J. Simões and B.S. Santos. "Requirements Engineering Using Mockups and Prototyping Tools: Developing a Healthcare WebApplication". In International Conference on Human Interface and the Management of Information, pp. 652-663. Springer. 2014.

[23] A.L. de Araújo, L. M. Cysneiros and V. M.B. Werneck. "NDR-Tool: Uma Ferramenta de Apoio ao Reuso de Conhecimento em Requisitos Não Funcionais”. In Workshop the Engenharia de software (WER 2014). Pucón, Chile, pp. 1-15. 2014.

[24] N. Larburu, R. G. Bults and H. J. Hermens. "Making medical treatments resilient to technological disruptions in telemedicine systems". In IEEE-EMBS International Conference on Biomedical and Health Informatics (BHI), pp. 285-288. IEEE. 2014.

[25] P. Loucopoulos, J. Sun, L. Zhao and F. Heidari. "A systematic classification and analysis of NFRs 19th Americas conference on information systems (AMCIS 2013)". Chicago, USA. AIS. 2013. 
[26] I. Hadar, P. Soffer and K. Kenzi. "The role of domain knowledge in requirements elicitation via interviews: an exploratory study". Requirements Engineering. Vol. 19 $\mathrm{N}^{\circ}$ 2, pp. 143-159. 2014.

[27] D. Ameller, C. Ayala, J. Cabot and X. Franch. "How do software architects consider nonfunctional requirements: An exploratory study". In 2012 20th IEEE International Requirements Engineering Conference (RE), pp. 41-50. IEEE. 2012.

[28] C. Rosenkranz, H. Vranesic and R. Holten. "Boundary interactions and motors of change in requirements elicitation: a dynamic perspective on knowledge sharing". Journal of the Association for Information Systems. Vol. $15 \mathrm{~N}^{\circ}$ 6, pp. 306. 2014.

[29] G. Salvendy and M.J. Smith. "Human Interface and the Management of Information. Interacting with Information". Symposium on Human Interface 2011, Held as Part of HCI International 2011, Orlando, FL, USA, July 9-14, 2011. Proceedings vol. LNCS 6772. Springer. 2011.

[30] J. Helming, M. Koegel, F. Schneider, M. Haeger, C. Kaminski, B. Bruegge, y B. Berenbach, "Towards a unified requirements modeling language," in Requirements Engineering Visualization (REV), 2010 Fifth International Workshop on, 2010, pp. 53-57. IEEE.

[31] X. Song, Z. Duan, y C. Tian, "NonFunctional Requirements Elicitation and Incorporation into Class Diagrams," in International Conference on Intelligent Information Processing, 2010, pp. 72-81. Springer

[32] S. Chakraborty, S. Sarker and S. Sarker, "An exploration into the process of requirements elicitation: a grounded approach". Journal of the Association for Information Systems. Vol. $11 \mathrm{~N}^{\circ}$ 4, pp. 212. 2010.

[33] B. Flores-Rios, O. Rodríguez-Elías y F. Pino. "Administración del producto de trabajo como gestión de conocimiento explícito de conformidad con el estándar ISO/IEC 15504". In CONISOFT, Xalapa, Veracruz, Mexico, pp. 29-36. 2013.
[34] T. Clancy. "The Standish Group CHAOS Report”. 2014.URL: https://www.projectsmart. co.uk/white-papers/chaos-report.pdf

[35] J. Garzás, F. J. Pino, M. Piattini and C. M. Fernández. "A maturity model for the Spanish software industry based on ISO standards". Computer Standards \& Interfaces. Vol. 35 $\mathrm{N}^{\mathrm{o}}$ 6, pp. 616-628. 2013.

[36] D. Minsal Pérez y Y. Pérez Rodríguez. "Hacia una nueva cultura organizacional: la cultura del conocimiento". Acimed. Vol. 16 $\mathrm{N}^{\circ}$ 3, 2007.

[37] M. Mendoza-Moreno, C. González-Serrano and F.J. Pino. "Focus group como proceso en ingeniería de software: una experiencia desde la práctica". Dyna. Vol. 80 N $^{\circ} 181$, pp. 51. 2013.

[38] S.L. Buitrón. "Marco de Trabajo para la elicitación de Requisitos No Funcionales basada en la Gestión de Conocimiento". Tesis para optar al grado de Magíster en Computación. FIET. Universidad del Cauca. Popayán, Colombia. 2016.

[39] M. Montesi and P. Lago. "Software engineering article types: An analysis of the literature". Journal of Systems and Software. Vol. 81 $\mathrm{N}^{\circ}$ 10, pp. 1694-1714. 2008.

[40] F.J. Pino, F. Ruiz, F. Garcia and M. Piattini. "A software maintenance methodology for small organizations: Agile_MANTEMA". Journal of Software: Evolution and Process. Vol. $24 \mathrm{~N}^{\circ}$ 8, pp. 851-876. 2012.

[41] E. Galvis-Lista y J. Sanchez-Torres. "Modelo de Referencia de Procesos de Gestión del Conocimiento para Organizaciones Desarrolladoras de Software de Colombia". 2014. URL: https://www.researchgate.net/ publication/266259125_Modelo_de_Referencia_ de_Procesos_de_Gestion_del_Conocimiento_ para_Organizaciones_Desarrolladoras_de_ Software_de_Colombia_V01.

[42] A. de J González, C.Z. Joaquí y C.A. Collazos. "Karagabi Kmmodel: Modelo de referencia para la introducción de iniciativas de gestión del conocimiento en organizaciones basadas en conocimiento". Ingeniare. Revista chilena de ingeniería. Vol. $17 \mathrm{~N}^{\circ}$ 2, pp. 223-235. 2009. 